\title{
The danger of trial-by-trial knowledge of results in perceptual averaging studies
}

\author{
BEN BAUER \\ Trent University, Oshawa, Ontario, Canada
}

\begin{abstract}
Revived interest in "intuitive statistics" (Peterson \& Beach, 1967) is evident in recent studies concerning the ability of observers to estimate mean size for ensembles of lines or of circles. To put the recent studies in context, and to highlight a potential danger in providing trial-by-trial knowledge of results (KOR), brief contact with previous research is made and a new experiment is presented demonstrating the malleability of responding to KOR. Together, these suggest two perils of KOR. First, given that the nature and even the existence of a molar size-averaging operator are both controversial, the proper criterion average for determining KOR is unclear. Second, whatever the operation observers use in this task, its scope and algorithm need to emerge from data unbiased by KOR. A corollary scaling concern is noted for averaging of areal stimuli (perceived as a compression function of physical area) as an instance of the more general concern that perceptual scaling factors must be taken into account when psychological averaging processes are studied.
\end{abstract}

There is renewed interest in, and vigorous debate about, the ability of human observers to compute statistical descriptors for properties of visual sets (see, e.g., Ariely, 2001; Chong, Joo, Emmanouil, \& Treisman, 2008; Chong \& Treisman, 2003, 2005a, 2005b; De Fockert \& Marchant, 2008; Emmanouil \& Treisman, 2008; Myczek \& Simons, 2008). The history of psychological research investigating this ability dates from the late 19th century (see, e.g., James's 1890 concept of "coalescence," and Messenger's 1912 concept of "constructive combination"). In the mid20th century, examination of the perceptual computations related to the mean (first moment) and variance (second moment) of ensemble properties continued as a particularly fruitful research area. For example, Helson's adaptation level theory (Helson, 1947; Helson, Michels, \& Sturgeon, 1954; Michels \& Helson, 1949; see also Brown, 1953; Parducci, Calfee, Marshall, \& Davidson, 1960) proposed that judgments for a given (current) stimulus are influenced by a weighted geometric mean of three values: that of the stimulus currently being judged; those of stimuli in the background (e.g., flankers and surrounds); and those of all prior stimuli. Thus, a running average of recent stimuli contributed to the instantaneous adaptation level of an observer interacting with the environment. Furthermore, the perceptual bisectioning work (e.g., Fagot \& Stewart, 1969; Pearson, 1922; Weene \& Held, 1966; Wolfe, 1923) can be seen as a type of averaging (Weiss \& Anderson, 1969).

Many classic intuitive averaging studies using ensembles of more than two items date from the 1960s and 1970s (e.g., Anderson, 1968; Laestadius, 1970; Spencer, 1961, 1963). Also, ensemble statistics were central to An- derson's (1970) functional measurement theory. Pickett (1964) used language curiously similar to that of current researchers, in suggesting that the "size of the texture 'population' should affect a statistical inference about it" (p. 14), and his discussion of ensemble subsampling anticipates some of the points raised in Myczek and Simons (2008; see also Simons \& Myczek, 2008) concerning the scope of visual operators. In brief, these later authors provided evidence that, in the case of perceived size, a strategy of subsampling a display can actually produce responses as good as or better than those from putative global (displaywide) averaging. This contention has added to the intensity of the current research on perceptual size averaging. A brief survey of some key studies in perceptual statistical research will help put these issues into perspective and provide the motivation for the issue addressed in the present experiment.

Peterson and Beach (1967) reviewed the research on perceptual averaging and other statistical computations, and their article "Man as an intuitive statistician" predates many additional investigations and reviews (Cosmides \& Tooby, 1996; Pollard, 1984; Slovic, Fischhoff, \& Lichtenstein, 1977). In many of these investigations, human observers were asked to inspect visual ensembles (also hefted weights, tones, odors, etc.) and to estimate the value of a requested statistic. In general, the observers were able to estimate central tendency values, variances, proportions, and probabilities for stimuli such as ensembles of 20 two-digit numbers, spatial locations, and moving objects. Performance approached normative in many cases, but there were also systematic deviations. Thus, there is a rich base of research suggesting that averages and vari- 
ance measures, at least, are computed groupwide by the sensory system (but see Myczek \& Simons, 2008, for an alternative view of size averaging). Forty years later, Juslin, Winman, and Hansson (2007) have suggested that it is time for "re-evoking the intuitive statistician" (p. 678), and the recent flurry of related activity in this journal and others seems to indicate that such a process is ongoing in visual perceptual averaging of size.

There is continuing concern about the nature of the $a v-$ erage that observers produce when asked. The reviews by Peterson and Beach (1967) and Pollard (1984) suggested that observers can produce averages consistent with the arithmetic mean - as opposed to, for example, the harmonic, geometric, or quadratic mean (root mean square [RMS]) - but other measures of central tendency could be educed, according to the demands and feedback given by the experimenter. For example, Peterson and Miller (1964) were able to show that observers can produce a mode, median, or mean (of speeds) as requested, according to a biasing payoff schedule (see also Massaro, 1969, for a demonstration of how observers will conform responses to feedback). A related ensemble statistic, numerousness (perceived number of items as a function of numerosity), can also be strongly influenced by feedback (e.g., Izard \& Dehaene, 2008; Minturn \& Reese, 1951). One benefit of the feedback is to reduce variability in the dependent measure. However, to the extent that the feedback influences the point estimator of the psychological value (e.g., the mental mean), or even the very process by which an observer arrives at the value (averaging or otherwise), it may influence the outcome in undesirable ways. This would cloud the investigation of perceptual averaging - whether it exists, its scope, its algorithm, and its vulnerability to bottom-up factors such as stimulus variability (see Chong \& Treisman, 2003; Laestadius, 1970; Spencer, 1961) and top-down factors such as attention (see Chong \& Treisman, 2005a; De Fockert \& Marchant, 2008).

The present article investigates the influence of knowledge of results (KOR) on the mean value, inferred from observers' responses as they calibrated to the KOR. For each of three groups of observers who received essentially the same ensembles, the KOR provided on each trial was based on a different criterion computation. For one group, the criterion for feedback, either positive (a small tilde) or negative (an " $x$ " on the screen), was the proximity of the terminal setting of a probe line to the arithmetic mean of the ensemble. For the second group, the criterion was the harmonic mean; for the third group, the criterion was the RMS (quadratic mean). Systematic changes in responding toward the decreed average would demonstrate that the process observers use when asked to indicate a mental mean is penetrable by KOR (at least for the psychophysical method and stimulus set used here). The inference would be that if there is a molar size-averaging operator, KOR should be avoided until its characteristics are better known. At the same time, if the operator's scope is limited to only a small number of items, or some other kind of processing is driving observers' responses (as suggested in Myczek \& Simons, 2008, and Simons \& Myczek, 2008), identification of the operations would clearly benefit from an unbiased experimental context.

Some recent investigations of perceptual size averaging using various psychophysical techniques (i.e., Chong et al., 2008; Chong \& Treisman, 2003, 2005a, 2005b; De Fockert \& Marchant, 2008; Emmanouil \& Treisman, 2008) have included KOR after judgments according to the arithmetic mean. The assumption is that the mental operation computes a measure very much like the arithmetic mean of the ensemble sizes. However, in essence, the arithmetic mean has become an independent variable, on the basis of which observers' correctness on a given trial is determined. The nature of the perceptual average (or whatever operation determines responding) should in fact be inferred from the dependent measures. Moreover, De Fockert and Marchant required at least 60\% accuracy in the third of three practice blocks for observers to be admitted to the recorded experimental trials. KOR was given (only on the three practice blocks) according to the arithmetic mean of diameters. Here, the tacit assumption is the existence of an arithmetic mean operator with a scope large enough to process a $12^{\circ} \times 12^{\circ}$ (visual angle) ensemble with diameters taken as input for the averaging of areal (circle) stimuli. The task was to indicate which of two probe circles shown after the ensemble was equivalent to the mean size of the ensemble.

Clearly, the precise determination of the mental computation behind supposed size averaging is a very difficult experimental problem, given the number of perceptual and cognitive biases involved in psychophysical measurement (e.g., size-brightness illusion; Ebbinghaus illusion; recency effects; regression effects; response category equalization; contrast and assimilation). These factors may result in systematic and random errors of sufficiently large magnitude to undermine definitive rejection of certain candidates for the mental computation. That said, it is worth approaching this question empirically, at least to exclude certain computations to the extent that experimental error and replication permit. To demonstrate that KOR may potentially contaminate results, the present experiment builds on the foundational work of Miller and Sheldon (1969), who investigated averaging for the continua of line length and of tilt (inclination), and on that of Weiss and Anderson (1969), who used an adjustment method for length averaging.

In Miller and Sheldon (1969), observers viewed ensembles of six lines of variable lengths (or inclinations) and provided numerical estimates of the average length (inclination) of the ensemble. The instructions to observers in the length averaging condition contained the following statements: "Your job will be to look over the six lines and to judge how long they are on average. . . . You are not expected to calculate the average by arithmetic ... just guess as accurately as possible their average length" (pp. 17-18). Also emphasized in the instructions was the requirement to produce the arithmetic mean of the lengths/inclinations. Observers' magnitude estimates for average length/inclination of six-item ensembles increased linearly (slopes near 1.0) with the arithmetic mean of the length/inclination of the ensemble. In a subsequent 
investigation, Miller, Pedersen, and Sheldon (1970) again emphasized the arithmetic mean to observers and found arithmetic averaging. Stanley (1974) replicated the Miller and Sheldon finding of near-nominal arithmetic averaging of visually presented angle pairs but with no mention of requiring a particular mean in the procedure section of their article. Weiss and Anderson (1969) had also found near-nominal line-length (arithmetic) averaging using the method of reproduction (adjustment) with both simultaneous and serial presentation of the to-be-averaged stimuli. Weiss and Anderson made it clear "that Ss were instructed to 'average,' but they were under no constraint about how they averaged. There are many measures of central tendency, and each S was free to choose his own" (p. 61). They did note a recency effect, in that stimuli presented at the end of the serial ensemble overcontributed to the produced mean.

The present experiment used a method similar to Weiss and Anderson's (1969), in that observers viewed an ensemble of lines and adjusted a probe item to indicate their mental average. No particular mean computation was implied by the instructions to observers. Ensembles of 16 lines were used here: four instances of four different line sizes. As indicated above, KOR according to three different criteria (between groups) followed each trial. Here, the choice of the specific KOR criteria is arbitrary, given the large number of central tendency measures. However, the work of Chong and Treisman (2003), Dakin (1997), and Miller and Sheldon (1969) has implicated a computation close to the arithmetic mean. The harmonic mean is systematically smaller than the arithmetic mean, and the RMS is larger. Using these two provides central tendency values on both sides of the arithmetic mean.

To the extent that the feedback alters the responses produced by observers across the course of the trials, the inference is that the process that leads to the responses (e.g., wholesale or subsample processes) is being subverted by training toward the mean consistent with the KOR. From another perspective, this experiment is designed to provide a definitive demonstration of arbitrary KOR determining the outcome of the experiment in favor of the prejudged mental average. An additional concern supporting the proscription of KOR in perceptual averaging studies is presented following the experiment.

\section{METHOD}

\section{Observers}

The observers were 66 students from three university campuses. They received $\$ 8$ or course credit for approximately $90 \mathrm{~min}$ of testing.

\footnotetext{
Apparatus

Timing, stimulus presentation, and response collection were performed using three iMac SE PPC750 computers with built-in 15-in. CRT monitors set to $1,024 \times 768$ pixel resolution at $75 \mathrm{~Hz}$. The controlling software was written using the VideoToolbox routines (Pelli, 1997). All screen changes were synchronized to the vertical refresh. Responses were collected via Apple M2452 keyboards. Viewing distance was approximately $50 \mathrm{~cm}$ (unrestrained), resulting in approximately $0.03^{\circ}$ of visual angle per pixel. In a dimly lit lab room, 1 to 4 observers were tested at a time.
}

Table 1

Line Widths and Ensemble Means

\begin{tabular}{|c|c|c|c|c|}
\hline \multirow[b]{2}{*}{ Ensemble } & \multirow{2}{*}{$\begin{array}{l}\text { Line Width } \\
\text { in Pixels }\end{array}$} & \multicolumn{3}{|c|}{ Mean } \\
\hline & & Arithmetic & Harmonic & RMS \\
\hline 1 & $12,20,28,40$ & 25.00 & 20.61 & 27.06 \\
\hline 2 & $16,24,34,50$ & 31.00 & 26.05 & 33.50 \\
\hline 3 & $20,28,40,60$ & 37.00 & 31.40 & 39.95 \\
\hline 4 & $24,32,46,70$ & 43.00 & 36.72 & 46.41 \\
\hline
\end{tabular}

\section{Stimuli and Trial Events}

Ensemble and probe items were horizontal lines of various widths (see Table 1), all 4 pixels in height. There were four different ensembles, each containing four line sizes, presented in equal quantities to create the 16-element ensembles. These sizes were selected so that the entire array could be seen with little or no head movement, to allow the three target means to be at least numerically no closer than 2 pixels, and because they are similar to the diameters of the circle stimuli used by Chong and Treisman $(2003,2005 a, 2005 b)$ in their perceptual averaging experiments.

The ensembles were presented roughly centered on the midpoint of the screen. The individual lines were positioned in the cells of an imaginary regular $4 \times 4$ grid with approximately 100 pixels between adjacent cells vertically and horizontally. Randomly selected offsets of $-6,0$, and +6 pixels in the vertical and horizontal directions were applied to each item to break up the regularity of the array. Because the assignment of the four instances of the four different sizes per ensemble to the 16 cells in the imaginary array was random on each trial, horizontal or vertical runs of a given size could occur. The jitter was introduced to reduce the probability of these runs lining up in the ensemble and perhaps being processed groupwise. Myczek and Simons (2008) achieved a similar goal by presenting their ensembles in the cells of a nonuniform imaginary grid. No attempt was made to control density.

The probe line was presented below the ensemble and its position jittered horizontally, randomly, on a range of \pm 42 pixels in steps of 6 pixels from the horizontal midpoint. The vertical placement jitter was up to \pm 12 pixels in steps of 6 from a point sufficiently low on the screen for the probe to be separate from the ensemble. Observers adjusted the width of the probe line using the arrow keys. The jitter in the probe line placement was introduced to avoid systematic space errors or bias (e.g., Brodie \& Dunn, 2005; Masin \& Agostini, 1991; Patston, Corballis, Hogg, \& Tippett, 2006).

The starting width for the probe line was determined on each trial according to the following algorithm: The width of the probe was based on the smallest ensemble member plus 1 pixel, or the largest ensemble member minus 1 pixel, with a random \pm 2 pixel adjustment. There were equal numbers of trials on which the starting value was near the minimum and near the maximum. For example, the small probes for Ensemble 3 were 19 or 23 and the large probes for Ensemble 3 were 57 or 61 .

Each observer completed 144 trials: 18 replications of the conditions 4 (ensembles) $\times 2$ (high vs. low probe start). Trial order was randomized for each observer. On each trial, the ensemble was displayed alone for $493 \mathrm{msec}$, at which point the accompanying probe line appeared. Both the ensemble and the probe remained on the screen until the observer had completed adjusting the probe line and pressed the space bar to end the trial. Observers adjusted the probe width using the left arrow key to reduce the width and the right arrow key to increase the width. At all times, the screen background was kept as a slightly warm neutral color. Stimuli were presented in white.

\section{Procedure}

Once the observer was seated in front of the computer, oral instructions on the use of the task and response keys were given and repeated on the screen when the program started. 
You will see displays containing a set of 16 lines of various sizes. Below the set of lines, you will see a single line. Please look at the set of 16 lines and estimate the average of the line sizes. Set the size of the single line to the average size. Do this by using the left arrow to make the line smaller and the right arrow to make the line longer. When the line is set to the group average of the 16 sizes, press spacebar for the next display.

As in Weiss and Anderson (1969), no particular measure of central tendency was emphasized.

A demonstration trial was shown, and then the KOR instructions were displayed. "After each trial, you will see: if your setting is close to the average, or $\mathbf{x}$ if your setting is far from the average." Observers were then reassured that people were quite good at this task and that the tolerance on the KOR was set quite close to the exact average, so they should not be worried if they saw a fair number of " $x$ "s early in the experiment. The KOR tolerance was set to approximately $\pm 10 \%$ (within integer math) of the target means, which were the arithmetic mean, the harmonic mean, and the RMS for 22 observers each.

\section{RESULTS}

The unit of analysis is the terminal setting of the probe line in pixels as a function of the ensemble and KOR provided. For each observer, a tally was made of the settings produced for each ensemble. From these tallies, a cumulative relative frequency table was generated. For each of these tables, the point of subjective equality (PSE) was estimated by probit analysis using psignifit Version 2.5.6 (Wichmann \& Hill, 2001). The PSE is considered to be the mental mean because the observer cannot tell the difference between the subjective mean of the ensemble and the probe size set. Figure 1 shows the cumulative relative frequency plots taken over all observers per KOR group.

Observer-level PSEs were then submitted to an ANOVA with two independent variables: KOR type (3 levels, between) and ensemble (4 levels, within). This analysis revealed a marginal main effect of $\operatorname{KOR}[F(2,60)=2.52$, $\left.M S_{\mathrm{e}}=49.6, p<.09\right]$ and no interaction of KOR with ensemble $(F<1)$. The main effect of ensemble was significant $\left[F(3,189)=918.6, M S_{\mathrm{e}}=4.18, p<.001\right]$. The mean terminal settings were $25.6(S D=3.3), 31.7(S D=3.3)$, $37.7(S D=4.3)$, and $43.3(S D=4.9)$ for Ensembles 1, 2, 3, and 4 , respectively. The excellent correspondence between these values and the actual arithmetic means in Table 1 should be ignored for two reasons. First, the marginal main effect of KOR indicates that there are likely differences among the terminal settings for the three KOR groups, and in fact, an analysis of KOR conditions RMS and arithmetic mean shows a main effect of $\operatorname{KOR}\left[F(1,42)=4.9, M S_{\mathrm{e}}=\right.$ $35.9, p<.04]$. Second, comparison of the data across all 144 trials per observer may mask learning effects as observers calibrate their responses to the KOR.

Therefore, the data were split into two blocks, with the first 72 trials in Block 1 and the last 72 trials in Block 2. The logic is as follows: If any of the three KOR criteria approximate the perceptual mean, the means (PSE settings) produced should change little as a function of block (i.e., no significant main effect or interaction of block with ensemble), because exposure to the KOR is merely confirm- ing what the observer is doing naturally and no change in behavior is required to receive positive KOR. This is the ideal case. It is also possible that a main effect or interaction of block could obtain simply due to fatigue, attention lapse, or practice with the equipment. This should occur with no consistent direction change in the settings across ensemble, because the KOR does not push observers to consistently bias their responses upward or downward across the different ensembles. Of course, the assumption here is that the learning/calibration in response to the KOR takes more than a few dozen trials to stabilize and would be detectable from Block 1 to Block 2. Statistically, if the KOR is altering observers' settings, there should be a main effect of block, or an interaction of block with ensemble, because the KOR is causing observers to calibrate their responses.

An ANOVA including the block factor (see the Appendix for mean PSEs and $S D$ s by KOR group, ensemble, and block) produced the following results. For the arithmetic mean KOR, there was no effect of block $(F<1)$, with PSEs changing by only 0.1 from Block 1 ( $M=33.6$, $S D=7.5)$ to Block $2(M=33.5, S D=7.0)$. Of course, ensemble produced a main effect $\left[F(3,63)=271, M S_{\mathrm{e}}=\right.$ 8.7, $p<.001]$, with overall PSEs of $24.9(S D=2.8)$, $30.8(S D=2.9), 36.5(S D=3.5)$, and $41.9(S D=4.7)$, for Ensembles 1, 2, 3, and 4, respectively. The block $\times$ ensemble interaction was not significant $[F(3,63)=1.25$, $\left.M S_{\mathrm{e}}=4.3, p>.3\right]$.

For the harmonic mean KOR, there was a main effect of block $\left[F(1,21)=9.4, M S_{\mathrm{e}}=21.3, p<.01\right]$, with PSEs dropping on average by 2.1 pixels from Block 1 to Block 2 . Again, ensemble produced a main effect $[F(3,63)=199.4$, $\left.M S_{\mathrm{e}}=7.1, p<.001\right]$. The block $\times$ ensemble interaction was significant $\left[F(3,63)=20.3, M S_{\mathrm{e}}=4.6, p<.01\right]$. This interaction is important, because the KOR is resulting in a slope difference (as a function of ensemble) across block. Assume for a moment that the arithmetic mean is truly the perceptual mean; in the early trials of Block 1, the observer would respond according to the arithmetic mean, but would be experiencing primarily negative KOR and trying to calibrate to the KOR. However, over the four ensembles used here, there is a slope difference between the growth of the arithmetic mean and the harmonic mean as a function of ensemble arithmetic mean (or sum). Obviously, the slope of arithmetic mean with respect to arithmetic mean is 1 , but the slope of the harmonic mean is shallower (0.90). Inspection of Table 1 reveals that, from Ensemble 1 to Ensemble 4, the arithmetic mean changes by 18 , whereas the harmonic mean change is only 16 . Thus, to change from an arithmetic mean responding mode to a harmonic mean responding mode requires setting the probe line smaller than expected, and continuing to do so as the ensemble mean gets larger. Figure 2 shows the slope differences for the harmonic mean and RMS plotted against the arithmetic mean of the ensemble.

For the group that received KOR based on the RMS, there was a main effect of block $\left[F(1,21)=5.6, M S_{\mathrm{e}}=\right.$ $30.7, p<.03$ ], with PSEs dropping by 2.0 across blocks. Ensemble produced a main effect $[F(3,63)=293.3$, 

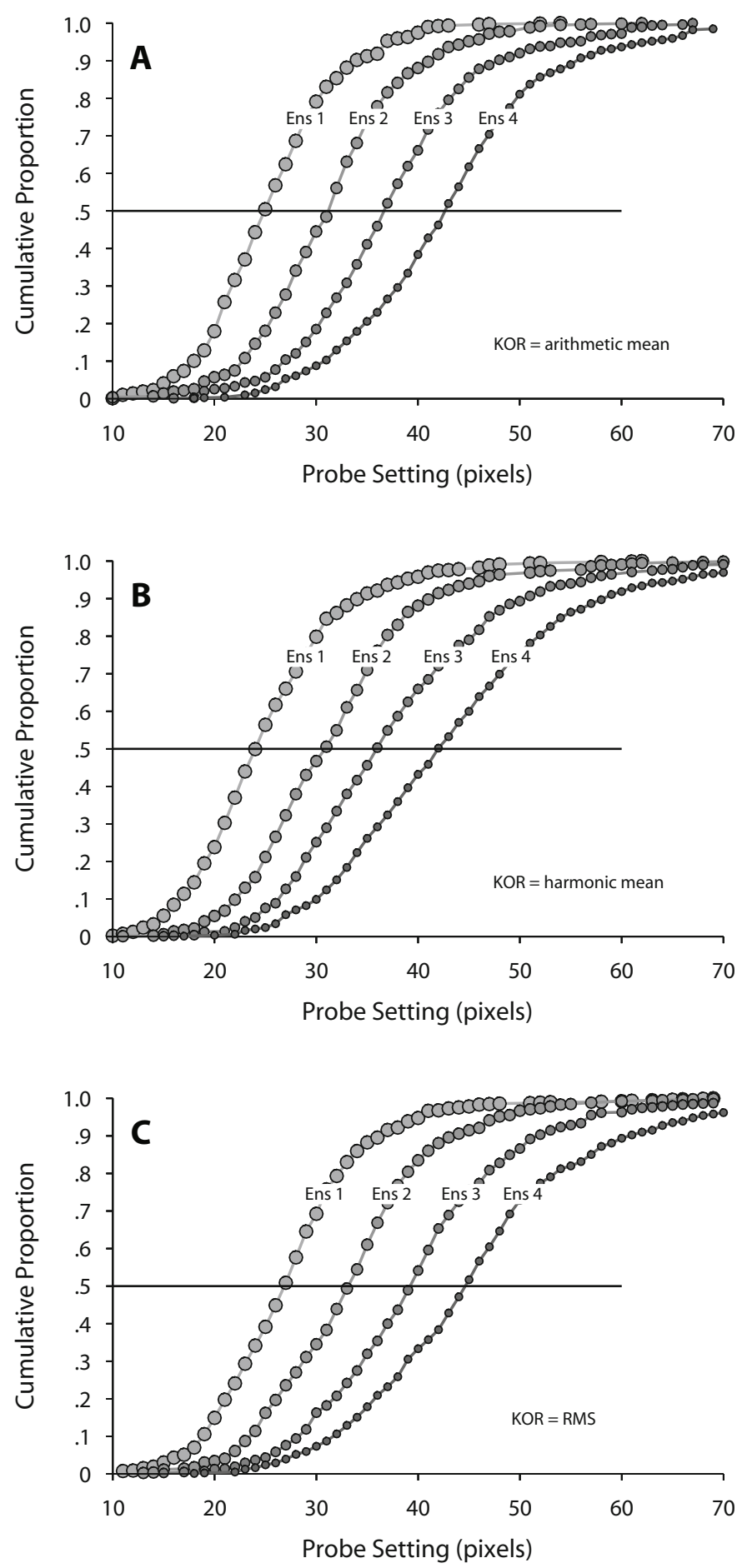

Figure 1. Relative cumulative frequency ogives for the three knowledge of results (KOR) groups. Points of subjective equality (PSEs) were estimated for each observer from analogous curves for each ensemble (Ens).

$\left.M S_{\mathrm{e}}=9.1, p<.001\right]$. Finally, the block $\times$ ensemble interaction was significant $\left[F(3,62)=4.5, M S_{\mathrm{e}}=4.6, p<\right.$ $.01]$. As seen in Figure 2, the slope of the RMS function is 1.08 with respect to the arithmetic mean.

Another way to look at the results is in terms of the deviation of settings (i.e., the PSEs) from the KOR mean over the course of the 144 trials. The data were separated into nine blocks of 16 trials each. On each trial, the target mean for the ensemble was subtracted from the probe setting and this deviation was then divided by the ensemble mean. These signed proportions were then converted to percents and averaged over the 16 trials per block. (Per- 


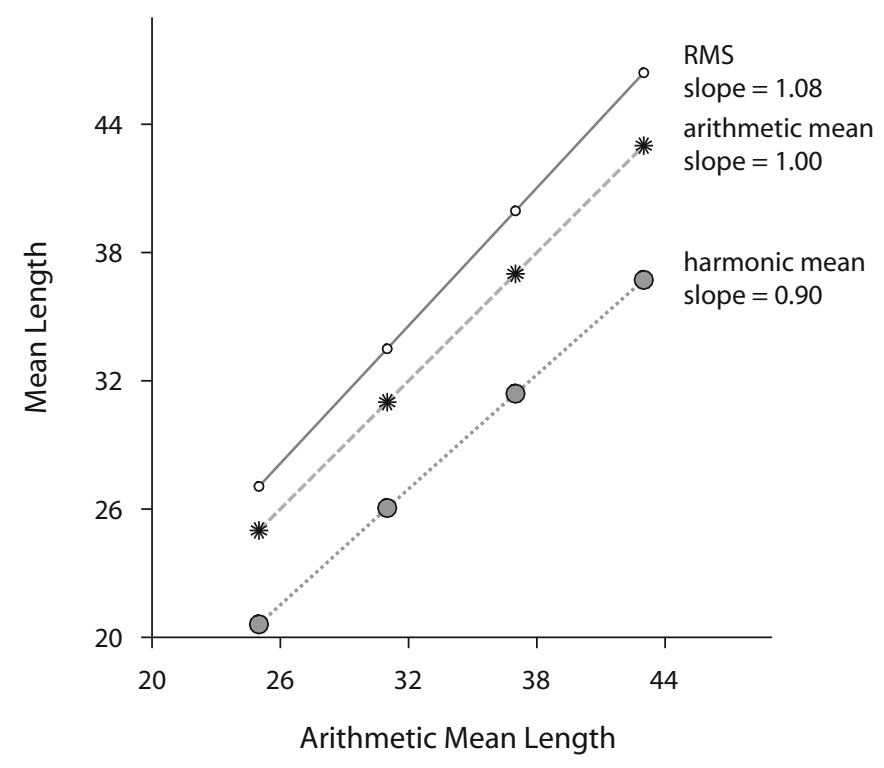

Figure 2. Slope differences in arithmetic mean, harmonic mean, and root mean square (RMS) over the range of ensembles used in the present experiment.

cents rather than absolute pixel deviations were used because there were variable numbers of trials of each ensemble within blocks of 16.)

The average percent deviations from target mean as a function of block are plotted in Figure 3. If observers had produced the settings strictly according to the KOR, all three curves would be flat running across the $0 \%$ deviation line (the dotted horizontal line in Figure 3). The departures from the $0 \%$ deviation line provide insight into the mental means computed.

Both the harmonic mean and RMS KOR groups give obvious evidence of a training effect. The RMS KOR group

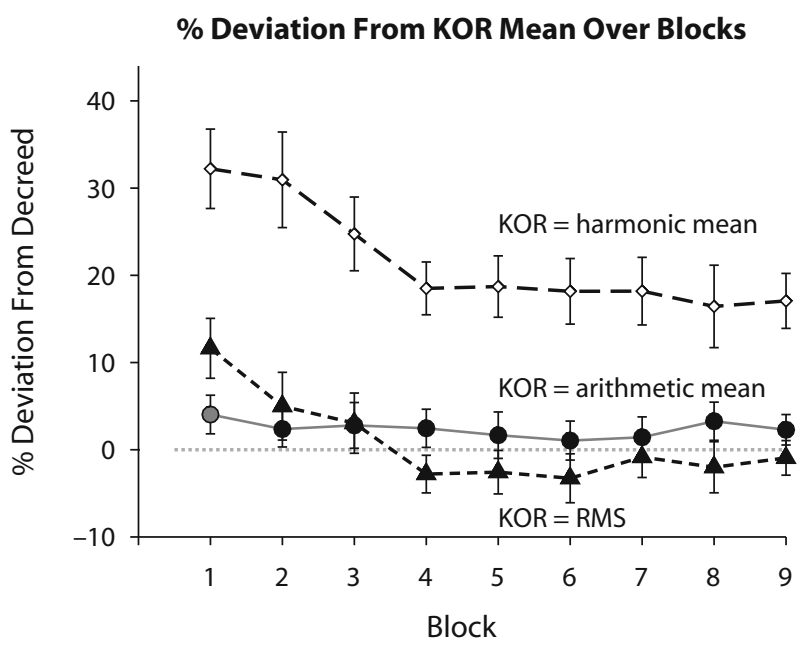

Figure 3. Average percent deviation from decreed mean as a function of block in Experiment 1. The horizontal dotted line represents performance based exactly on the knowledge of results (KOR) mean. first produced settings too large for this mean but calibrated quickly toward the RMS with a slight undershoot in the direction of the arithmetic mean. From Table 1, it can be seen that the arithmetic mean is 2.1-3.4 pixels smaller than the RMS across ensembles. This amounts to a $-8 \%$ difference. From Blocks 4 to 9 , the probe settings for RMS KOR were approximately $-2 \%$ from the true RMS, or about $6 \%$ above the arithmetic mean. Interestingly, the line for the arithmetic mean is essentially horizontal (no effect of block for the arithmetic mean KOR condition, $F<1$ ), with an average setting of $+2.3 \%$ above the true arithmetic mean. Across ensembles, the range of line widths was $12-70$ pixels. This $+2.3 \%$ error amounts to a maximum of 1.6 pixels or $0.05^{\circ}$ of visual angle. Given that the arithmetic mean was within the KOR tolerance for the RMS (i.e., setting the probe to the arithmetic mean would result in positive KOR), it is telling that observers learned to set the probe line a few pixels too small for the RMS. Essentially, if they anchored to the arithmetic mean but learned to set slightly high, they could do well (in terms of receiving positive feedback) under RMS KOR.

For the harmonic mean KOR group, setting to the arithmetic mean would not work (in terms of receiving positive feedback), because the arithmetic mean is not inside the harmonic mean tolerance. The arithmetic means are between 4.4 and 6.3 pixels larger than the harmonic means, amounting to about $19 \%$. Interestingly, in the harmonic mean KOR condition, the average deviation of the settings from the harmonic mean is $21.7 \%$. The observers apparently calibrated their settings to the arithmetic mean, and adjusted upward to obtain the positive KOR.

The implications are obvious. When given KOR according to the arithmetic mean, observers do not appear to adjust their strategy over the course of 144 trials. When given bogus KOR, attempting to force the mental mean 
too high or too low, observers base their settings on an adjusted arithmetic mean.

Of course, the settings in the arithmetic mean KOR group slightly overshoot the arithmetic mean. This may be an example of the numerosity heuristic (tendency of observers to overestimate properties as a function of numerosity; see Krueger, 1970; Pelham, Sumarta, \& Myaskovsky, 1994) or a case of a slight deviation of the Stevens exponent from 1 . If the exponent is less than 1 , smaller lines are overestimated in size and larger ones underestimated. This means that the overestimation should decrease with ensemble mean. If the exponent is greater than 1 , overall overestimation will occur, but will increase with ensemble size. Given that the systematic overestimation amounts to only about $2 \%$ or 0.3 to 1.4 pixels across the widths tested, such an analysis would be pushing the limits of the precision in the data. The data, taken at face value, support the explanation based on a Stevens exponent of slightly less than 1 , because overestimation declines with absolute ensemble mean, and the log-log fit of geometric mean setting with ensemble arithmetic mean produces a slope (Stevens exponent) of 0.96 .

\section{DISCUSSION}

The critical result is that observers in the arithmetic mean KOR group did not change behavior in any noteworthy systematic way across trials. Their performance approximates the arithmetic mean as early as the first block. Observers in the two other KOR conditions show a substantial learning effect that asymptotes after Block 4 as close to the arithmetic mean as permitted by the tolerance on the KOR. The implication is that the arithmetic mean is a good approximation to the mental mean computation, assuming displaywide averaging of sizes. Note, however, that the present experiment does not allow a conclusion that the perceptual mean algorithm involves the sum of sizes divided by the item count. This caveat is offered for several reasons. First, there are other numerical ways to compute a value that would look very much like the arithmetic mean. Second, Myczek and Simons (2008) and Simons and Myczek (2008) contend that observers may not in fact be computing a measure of central tendency based on displaywide parallel processing of the items; rather, they suggest that subsampling the display could result in behavior that mimics global averaging. Third, as mentioned above, the experimental precision required to definitively determine the algorithm is difficult to obtain. At the same time, it does appear that the mental computation may produce something akin to the arithmetic mean (perhaps with a constant) at least over the range of stimuli used here. It is possible to use Fagot's (1963) generalized power law

$$
\bar{\Psi}=\left[\frac{1}{n} \sum_{i=1}^{n} \Phi_{i}^{k}\right]^{\frac{1}{k}}
$$

to interrogate the data. ${ }^{1}$

In this equation, $\bar{\Psi}$ is the mental mean, $\Phi_{i}$ are the physical lengths of the stimuli, $n$ is the item count (ensemble size), and $k$ is the value to solve for. As $k$ approaches 0 , the mental mean approximates the geometric mean. A $k$ value near 1 implicates the arithmetic mean. Essentially, solution of this equation indicates the nature of the psychophysical scaling of the ensemble members that would be required to produce an obtained psychological mean. As a trivial example, if an ensemble had just two numeric values, 6 and $10, n=2$, and the $\bar{\Psi}=8.0$, we could solve for $k$ and discover that

$$
8=\left[\frac{1}{2}\left(6^{1}+10^{1}\right)\right]^{\frac{1}{1}},
$$

which indicates an arithmetic mean. If $\bar{\Psi}$ were smaller than 8.0 (e.g., nearer to the geometric mean of 7.75), $k \rightarrow 0$. As $\bar{\Psi}$ increases toward the RMS of $8.25, k \rightarrow 2$, which follows because

$$
8.25=\left[\frac{1}{2}\left(6^{2}+10^{2}\right)\right]^{\frac{1}{2}}=\sqrt{\frac{136}{2}} .
$$

Substituting each ensemble's members into $\Phi_{i}$ and the corresponding empirical means (PSEs) into $\bar{\Psi}, k$ averages 0.980 for the arithmetic mean KOR with all trials included. For the two other KOR conditions, $k$ is computed only on the basis of the previous 72 trials, because the major effect of training has subsided by then. For the harmonic mean KOR condition, $k=0.527$, and for the RMS KOR data, $k=1.309$. Two conclusions can be drawn from these values. First, and most critically for the present purposes, KOR can dramatically change the inferred mental mean. Second, given that performance was essentially flat across blocks in the arithmetic mean KOR condition (see Figure 3), and average $k$ is very close to 1 across ensembles (but see below), something similar to the arithmetic mean may be computed. However, this last statement needs to be qualified, especially because of the limited stimulus range used and the fact that the individual $k$ values show a trend toward decreasing as ensemble arithmetic mean increases (see Figure 4). Let us remember that these fits involve minimizing the deviation of an empirical value with an exponent from a sum of physical measurements, each with that same exponent. Even with the tight correspondence of the PSEs (Ensemble $1=$ 25.19 , Ensemble $2=31.09$, Ensemble $3=36.84$, Ensemble $4=42.49$ ) to the physical means (Ensemble $1=$ 25.0, Ensemble $2=31.0$, Ensemble $3=37.0$, Ensemble $4=43.0$ ), the $k$ values look quite variable. In fact, the deviations from 1 (emphasized by the magnified scale of Figure 4) are less of a concern than is the systematic change in the PSEs from overestimation to underestimation of the physical arithmetic mean. This could reflect a response bias of some kind, or it might be a clue toward more precise specification of the mental mean algorithm. Furthermore, given that the theoretic expected value of $k$ for computations of the arithmetic mean is 1 , one would expect that, empirically, the observed $k$ values should vary unsystematically around 1 due to experimental error. The systematicity of the deviations from 1 suggest either that there are other factors unaccounted for in displaywide averaging, or that the assumption of displaywide averag- 


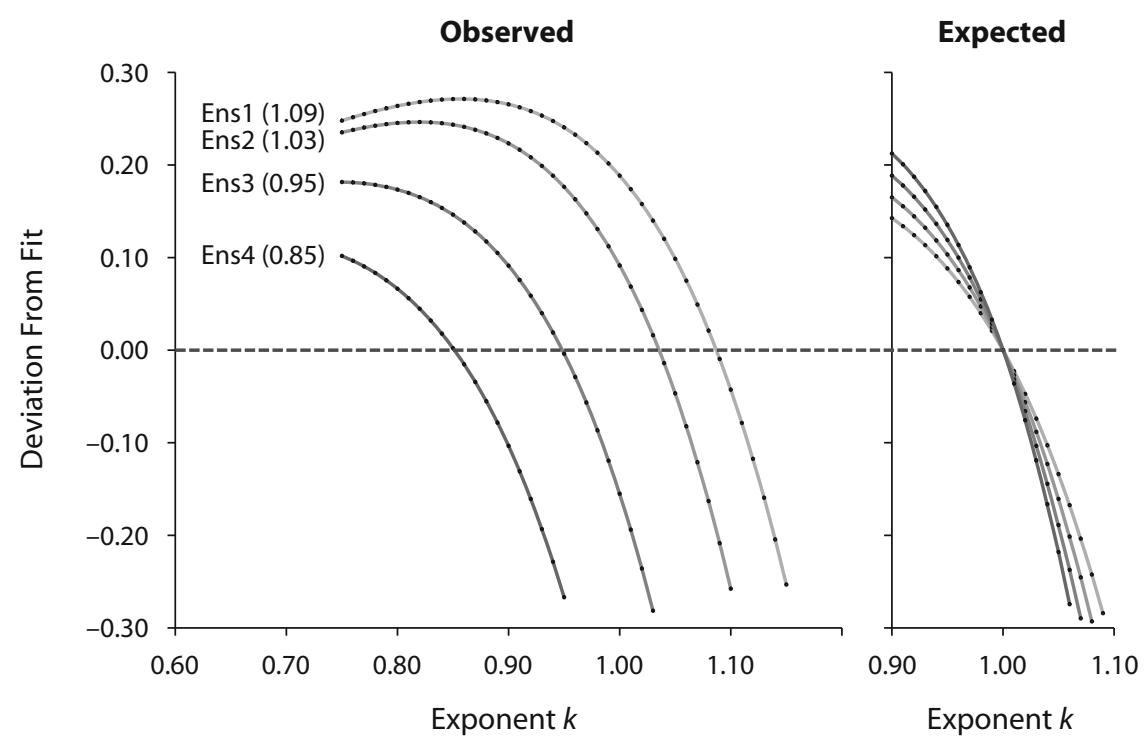

Figure 4. Deviation from fit according to $k$ as a function of ensemble for the observed psychological means (left panel) and expected arithmetic mean. For a true arithmetic mean, all ensembles should produce curves that cross the horizontal ( 0 deviation) line at the same $k$ value. Ens $=$ Ensemble. The values in parentheses are the obtained $k$ s.

ing is incorrect. Again, more research is required to make this determination.

The biggest concern about KOR, however, does not come from experiments using lineal stimuli. The KOR issue is relatively unimportant for lines, given that the present experiment suggests that, to a first approximation, the arithmetic mean may be close enough for the range of stimuli used here. Underlying this is the requirement that the mental representation of line length scale linearly with physical line length. Of course, this has been verified many times, with a variety of psychophysical procedures (e.g., Baird, Kreindler, \& Jones, 1971; Baird, Romer, \& Stein, 1970; Hartley, 1981; Hubbard, 1994; Kerst \& Howard, 1978; Markley, Ayers, \& Rule, 1969; Moyer, Bradley, Sorenson, Whiting, \& Mansfield, 1978; Rule, 1969; Stevens, 1969; Stevens \& Galanter, 1957; M. Teghtsoonian, 1965; M. Teghtsoonian \& R. Teghtsoonian, 1971; R. Teghtsoonian \& M. Teghtsoonian, 1970; Walker, 2002; Zwislocki, 1983; Zwislocki \& Goodman, 1980).

However, areal stimuli (filled or unfilled circles) are typically used in perceptual averaging experiments (e.g., Ariely, 2001; Chong et al., 2008; Chong \& Treisman, 2003, 2005a, 2005b; De Fockert \& Marchant, 2008; Emmanouil \& Treisman, 2008). It is well known that if observers are asked to react to the "size" as opposed to the area of circles or spots, the representation is not linear with the physical area and follows a compressive power function with an exponent $\beta$ (according to Stevens's power law) on the order of 0.70 to 0.80 (e.g., Algom, Wolf, \& Bergman, 1985; Chang, 1977; Crawford, 1971; da Silva, Marques, \& Ruiz, 1987; Ekman, 1958; Flannery, 1971; Griffen, 1985; Hubbard, 1994; Kemp, 1988; Krider, Raghubir, \& Krishna, 2001; Mashhour \& Hosman, 1968; Rule, Curtis, \& Markley, 1970; Stevens \& Galanter, 1957; Stevens \&
Guirao, 1963; M. Teghtsoonian, 1965; M. Teghtsoonian \& R. Teghtsoonian, 1971, 1983; Walker, 2002). Thus, small areas are overestimated and large areas are underestimated (see also Appendix A in Chong \& Treisman, 2003). Therefore, even if we accept the arithmetic mean as a good approximator of the mental mean, KOR cannot be based on the arithmetic mean of the diameters or areas because of the compression function (Stevens's exponent $<1$ ). KOR based on these could force observers out of spontaneous mental averaging (if that is what they are doing), and into a mode of calibration in response to the "correct/incorrect" feedback provided, as demonstrated above.

Consider the data in M. Teghtsoonian (1965) for magnitude estimates of "apparent size" of circles. The circles (judged one at a time) had radii from 1 to 9 inches in 1-in. increments. The reported $\beta$ was 0.76 . The scatterplot from which this exponent was computed is shown in Figure 1 of M. Teghtsoonian. The coordinates from this plot were recovered using g3data (see Bauer \& Reynolds, 2008) and entered into a regression of $\ln$ (estimates) on $\ln ($ area), where $\ln$ is the natural (Naperian) logarithm. This resulted in the equation $\ln (\Psi)=.759 \ln (\Phi)-0.89$; or, in linear space

$$
\Psi=e^{-0.89} \times \Phi^{0.759},
$$

with $\Phi$, in this case, in square inches. The exponent obtained with this method accords perfectly with M. Teghtsoonian's value. Now, take an ensemble containing four items using M. Teghtsoonian's stimulus sizes-for example, circles with radii of $1,3,7$, and 9 in. The average (arithmetic mean) of the radii is 5 , which corresponds to an area of $78.5 \mathrm{in}^{2}$. The areas of the circles $(3.14,28.27$, $153.94,254.47$ in. $^{2}$ ) produce a mean of $110 \mathrm{in.}^{2}$. The perceived sizes, according to Equation 2, are 0.97, 5.19, 
18.78, and 27.50, which appear small; however, the absolute magnitude of these is determined by the modulus (standard) used in the magnitude estimation procedure, which had a 5-in. radius, or an area of 78.5 in..$^{2}$ in this case. The arithmetic mean of the "apparent size" estimates is 13.11. Substituting this $\Psi$ value into $\Psi=e^{-0.89} \times \Phi^{0.759}$ and solving for $\Phi$ results in an area of 95.88 in. ${ }^{2}$. Which of these three values should be considered correct for the purposes of providing unbiased KOR? These vexing problems highlight the danger in providing KOR, or conversely, the importance of understanding the psychologically relevant stimulus properties. Fortunately, the stimuli used by Ariely (2001) and those in the various experiments by Chong and Treisman were not linearly spaced. Rather than adding a constant to get the next size up in an ensemble (to achieve equal physical scaling), these studies used a multiplicative constant that spaces the sizes out at approximately equal perceptual steps - or, at least, in suprathreshold steps. This foresight obviates many problems and, at least on an ordinal task (e.g., determining which of two displays has the bigger mean), the KOR may be valid with several measures of central tendency, at least according to an assumption of displaywide averaging.

Improperly set KOR also has the potential to inflate thresholds as calculated with probit analysis. Given that probit analysis reveals the value of the comparison stimulus at $\pm 1 S D$ (or at a slightly wider value in the case of Chong \& Treisman's work), and that observer calibration may require (in the present case) dozens of trials, this adds variance to the data, and will increase the band of stimuli inside the $\pm 1 S D$ limits. Perhaps more importantly, this may taint the experiment by placing the observer in a frustrating situation in which "intuitive" responding is signaled as incorrect. This assumes, of course, that Miller and Sheldon (1969) were correct when they concluded that "the 'power law' might be expected to apply to the SA [subjective average] when it holds for the unitary continuum" (p. 21). Weiss (1972), in an article titled "Averaging: An empirical validity criterion for magnitude estimation," also expressed the importance of further testing the generalization of Stevens's law to averaging; interestingly, however, the last 40 years have seen little additional evidence for this assertion. One noteworthy exception is Kuriki (2004), who demonstrated that observers can average colors. He provided no feedback to his observers and the colors used were properly scaled (using a psychologically based color space). The correspondence between the observers' average productions and the chromatic average of the colors in the ensembles was systematic and quite accurate. This experiment highlights the importance of correct psychological scaling in computation of the averages.

There is another potential danger in the KOR that arises in particular if the assumed global averaging process is not responsible for observers' decisions. Recall that Myczek and Simons (2008) have shown that subsampling the display could result in performance that mimics global averaging. In this case, KOR might be even more dubious in that, on the basis thereof, observers may learn particular strategies that satisfy the criteria by which the positive and negative feedback are computed. These strategies could be direct (such as computation of a value based on sampling a small number of items) or indirect anchor and adjust strategies, as suggested in the present data with the harmonic mean or RMS feedback. The algorithm used might even be unknown to the observer, since some forms of unintentional correlation between stimulus properties (e.g., overall luminance, interstimulus spacing, bounding box, etc.) are learned implicitly. ${ }^{2}$

In terms of investigating the averaging process and model building, it is crucial to determine - or at least to clearly state assumptions about - the physical property of the stimulus that is psychologically relevant, and its psychological representation. As Runeson (1994, p. 762) warned, "rather than prescribing the perceptual attributes, and trying to make people good meters of them, psychophysics must take as its primary goal to search for and identify the properties that are actually perceived."

\section{AUTHOR NOTE}

The author thanks D. J. Simons, Sang Chul Chong, and an anonymous reviewer for their insights and suggestions. Thanks also to Glenn Legault and Jason Kerr for help in collecting the data and to the observers for their patience and perseverance. Correspondence concerning this article should be addressed to B. Bauer, Department of Psychology, Trent University, P.O. Box 385, 2000 Simcoe Street North, Oshawa, ON, L1H 7L7 Canada (e-mail: benbauer@trentu.ca).

\section{REFERENCES}

Algom, D., Wolf, Y., \& Bergman, B. (1985). Integration of stimulus dimensions in perception and memory: Compositional rules and psychophysical relations. Journal of Experimental Psychology: Human Perception \& Performance, 114, 451-471.

Anderson, N. H. (1968). Averaging of space and number stimuli with simultaneous presentation. Journal of Experimental Psychology, 77, 383-392.

Anderson, N. H. (1970). Functional measurement and psychological judgment. Psychological Review, 77, 153-170.

ARIELY, D. (2001). Seeing sets: Representation by statistical properties. Psychological Science, 12, 157-162.

Baird, J. C., Kreindler, M., \& Jones, K. (1971). Generation of multiple ratio scales with a fixed stimulus attribute. Perception \& Psychophysics, 71, 399-403.

BAIRD, J. C., Romer, D., \& Stein, T. (1970). Test of a cognitive theory of psychophysics: Size discrimination. Perceptual \& Motor Skills, 30, 495-501.

BAuer, B., \& Reynolds, M. (2008). Recovering data from scanned graphs: Performance of Frantz's g3data software. Behavior Research Methods, 40, 858-868.

Brodie, E. E., \& DunN, E. M. (2005). Visual line bisection in sinistrals and dextrals as a function of hemispace, hand, and scan direction. Brain \& Cognition, 58, 149-156.

Brown, D. R. (1953). Stimulus-similarity and the anchoring of subjective scales. American Journal of Psychology, 66, 199-214.

Chang, K.-T. (1977). Visual estimation of graduated circles. Canadian Cartographer, 14, 130-138.

Chong, S. C., Joo, S. J., Emmanouil, T.-A., \& Treisman, A. (2008). Statistical processing: Not so implausible after all. Perception \& Psychophysics, 70, 1327-1334.

Chong, S. C., \& Treisman, A. (2003). Representation of statistical properties. Vision Research, 43, 393-404.

Chong, S. C., \& Treisman, A. (2005a). Attentional spread in the statistical processing of visual displays. Perception \& Psychophysics, 13, 1-13. 
Chong, S. C., \& Treisman, A. (2005b). Statistical processing: Computing the average size in perceptual groupings. Vision Research, $\mathbf{4 5}$, 891-900.

Cosmides, L., \& Toовy, J. (1996). Are humans good intuitive statisticians after all? Rethinking some conclusions from the literature on judgment under uncertainty. Cognition, 58, 1-73.

Crawford, P. V. (1971). Perception of grey-tone symbols. Annals of the Association of American Geographers, 61, 721-735.

DAKIN, S. C. (1997). The detection of structure in Glass patterns: Psychophysics and computational models. Vision Research, 37, 2227 2246.

da Silva, J. A., Marques, S. L., \& Ruiz, E. M. (1987). Subjective differences in exponents of psychophysical power functions for inferred, remembered, and perceived area. Bulletin of the Psychonomic Society, 25, 191-194.

De Fockert, J. W., \& Marchant, A. P. (2008). Attention modulates set representation by statistical properties. Perception \& Psychophysics, 70, 789-794.

EkMAN, G. (1958). Two generalized ratio scaling methods. Journal of Psychology, 45, 287-295.

Emmanouil, T.-A., \& Treisman, A. (2008). Dividing attention across feature dimensions in statistical processing of perceptual groups. Perception \& Psychophysics, 70, 946-954

FAGOT, R. F. (1963). On the psychophysical law and estimation procedures in psychophysical scaling. Psychometrika, 28, 145-160.

FAGot, R. F., \& Stewart, M. R. (1969). Individual half-judgment brightness functions. Perception \& Psychophysics, 5, 165-170.

FlanNeRY, J. J. (1971). The relative effectiveness of some common graduated point symbols in the presentation of quantitative data. $\mathrm{Ca}$ nadian Cartographer, 8, 96-109.

GRIFFEN, T. L. C. (1985). Group and individual variations in judgment and their relevance to the scaling of graduated circles. Cartographica, 22, 21-37.

Hartley, A. A. (1981). Mental measurement of line length: The role of the standard. Journal of Experimental Psychology: Human Perception \& Performance, 7, 309-317.

Helson, H. (1947). Adaptation level as a frame of reference for prediction of psychological data. American Journal of Psychology, 60, 1-29.

Helson, H., Michels, W. C., \& Sturgeon, A. (1954). The use of comparative rating scales for the evaluation of psychophysical data. American Journal of Psychology, 67, 321-326.

Hubbard, T. L. (1994). Memory psychophysics. Psychological Research, 56, 237-250.

Izard, V., \& Dehaene, S. (2008). Calibrating the mental number line. Cognition, 106, 1221-1247.

Juslin, P., Winman, A., \& Hansson, P. (2007). The naive intuitive statistician: A naive sampling model of confidence intervals. Psychological Review, 114, 678-703.

Kemp, S. (1988). Memorial psychophysics for visual area: The effect of retention interval. Memory \& Cognition, 16, 431-436.

Kerst, S. M., \& Howard, J. H. (1978). Memory psychophysics for visual area and length. Memory \& Cognition, 6, 327-335.

Krider, R. E., Raghubir, P., \& Krishna, A. (2001). Pizzas: $\pi$ or square? Psychophysical biases in area comparisons. Marketing Science, 20, 405-425.

Krueger, L. E. (1970). Apparent combined length of two-line and fourline sets. Perception \& Psychophysics, 8, 210-214.

KURIKI, I. (2004). Testing the possibility of average-color perception from multi-colored patterns. Optical Review, 11, 249-257.

LAESTADIUS, J. E. (1970). Tolerance for errors in intuitive mean estimations. Organizational Behavior \& Human Performance, 5, 121-124.

Markley, R. P., Ayers, D., \& Rule, S. J. (1969). Similarity judgments of line length. Perception \& Psychophysics, 6, 58-60.

Mashrour, M., \& Hosman, J. (1968). On the new "psychophysical law": A validation study. Perception \& Psychophysics, 3, 367-375.

Masin, S. C., \& Agostini, A. A. (1991). Attentional scanning and space errors. Perception \& Psychophysics, 50, 285-289.

Massaro, D. W. (1969). The effects of feedback in psychophysical tasks. Perception \& Psychophysics, 6, 89-91.

Michels, W. C., \& Helson, H. (1949). A reformulation of the Fechner law in terms of adaptation level applied to ratio scaling. American Journal of Psychology, 62, 355-368.

Miller, A. L., Pedersen, V. M., \& Sheldon, R. W. (1970). Magnitude estimation of average length: A follow-up. American Journal of Psychology, 83, 95-102.

Miller, A. L., \& SHeldon, R. W. (1969). Magnitude estimation of average length and average inclination. Journal of Experimental Psychology, 81, 16-21.

Minturn, A. L., \& ReEse, T. W. (1951). The effect of differential reinforcement on the discrimination of visible number. Journal of Psychology, 31, 201-231.

Moyer, R., Bradley, D., Sorenson, M., Whiting, J., \& Mansfield, D. (1978). Psychophysical functions for perceived and remembered size. Science, 200, 330-332.

MyczeK, K., \& Simons, D. J. (2008). Better than average: Alternatives to statistical summary representations for rapid judgments of average size. Perception \& Psychophysics, 70, 772-788.

Parducci, A., Calfee, R. C., Marshall, L. M., \& Davidson, L. P. (1960). Context effects in judgment: Adaptation level as a function of the mean, midpoint, and median of the stimuli. Journal of Experimental Psychology, 60, 65-77.

Patston, L. L. M., Corballis, M. C., Hogg, S. L., \& Tippett, L. J. (2006). The neglect of musicians: Line bisection reveals an opposite bias. Psychological Science, 17, 1029-1031.

Pearson, E. S. (1922). On the variations in personal equation. Biometrika, 14, 23-102.

Pelham, B. W., Sumarta, T. T., \& Myaskovsky, L. (1994). The easy path from many to much: The numerosity heuristic. Cognitive Psychology, 26, 103-133.

Pelli, D. G. (1997). The VideoToolbox software for visual psychophysics: Transforming numbers into movies. Spatial Vision, 10, 437-442.

Peterson, C. R., \& BeACH, L. R. (1967). Man as an intuitive statistician. Psychological Bulletin, 68, 29-46.

Peterson, C. R., \& Miller, A. (1964). Mode, median and mean as optimal strategies. Journal of Experimental Psychology, 68, 363-367.

PicketT, R. M. (1964). The perception of visual texture. Journal of Experimental Psychology, 68, 13-20.

POLLARD, P. (1984). Intuitive judgments of proportions, means, and variances. Current Psychological Research \& Reviews, 3, 5-18.

Rule, S. J. (1969). Subject differences in exponents from circle size, numerousness, and line length. Psychonomic Science, 15, 284-285.

Rule, S. J., Curtis, D. W., \& Markley, R. P. (1970). Input and output transformations from magnitude estimation. Journal of Experimental Psychology, 86, 343-349.

Runeson, S. (1994). Psychophysics: The failure of an elementaristic dream. Behavioral \& Brain Sciences, 17, 761-763.

Simons, D. J., \& MyczeK, K. (2008). Average size perception and the allure of a new mechanism. Perception \& Psychophysics, 70, 13351336.

Slovic, P., Fischhoff, B., \& Lichtenstein, S. (1977). Behavioral decision theory. Annual Review of Psychology, 28, 1-39.

SPENCER, J. (1961). Estimating averages. Ergonomics, 4, 317-328.

SPENCER, J. (1963). A further study of estimating averages. Ergonomics, 6, 255-265.

Stanley, G. (1974). Adding and averaging angles: Comparison of haptic-visual and visual-visual information integration. Acta Psychologica, 38, 331-336.

SteVens, S. S. (1969). On predicting exponents for cross-modality matches. Perception \& Psychophysics, 6, 251-256.

Stevens, S. S., \& Galanter, E. H. (1957). Ratio scales and category scales for a dozen perceptual continua. Journal of Experimental Psychology, 54, 377-411.

Stevens, S. S., \& Guirao, M. (1963). Subjective scaling of length and area and the matching of length to loudness and brightness. Journal of Experimental Psychology, 66, 177-186.

Teghtsoonian, M. (1965). The judgment of size. American Journal of Psychology, 78, 392-402.

Teghtsoonian, M., \& Teghtsoonian, R. (1971). How repeatable are Stevens's power law exponents for individual subjects? Perception \& Psychophysics, 10, 147-149.

Teghtsoonian, M., \& Teghtsoonian, R. (1983). Consistency of in- 
dividual exponents in cross-modality matching. Perception \& Psychophysics, 33, 203-214.

Teghtsoonian, R., \& Teghtsoonian, M. (1970). Two varieties of perceived length. Perception \& Psychophysics, 8, 389-392.

WALKER, B. N. (2002). Magnitude estimation of conceptual data dimensions. Journal of Experimental Psychology: Applied, 8, 211-221.

WeENE, P., \& HeLD, R. (1966). Changes in perceived size of angles as a function of orientation in the frontal plane. Journal of Experimental Psychology, 71, 55-59.

WeIss, D. J. (1972). Averaging: An empirical validity criterion for magnitude estimation. Perception \& Psychophysics, 12, 385-388.

WeIss, D. J., \& ANDERSON, N. H. (1969). Subjective averaging of length with serial presentation. Journal of Experimental Psychology, 82, 52-63.

Wichmann, F. A., \& Hill, N. J. (2001). The psychometric function: I. Fitting, sampling and goodness of fit. Perception \& Psychophysics, 63, 1293-1313.
Wolfe, H. K. (1923). On the estimation of the middle of lines. American Journal of Psychology, 34, 313-358.

ZWISLOCKI, J. J. (1983). Group and individual relations between sensation magnitude and their numerical estimates. Perception \& Psychophysics, 33, 460-468.

Zwislocki, J. J., \& Goodman, D. A. (1980). Absolute scaling of sensory magnitude: A validation. Perception \& Psychophysics, 28, 2838.

\section{NOTES}

1. The author thanks Peter Killeen for pointing out, in the context of an earlier version of the present article, the utility of Fagot's (1963) work in interpreting perceptual averaging results.

2. The author thanks D. J. Simons for pointing out this important concern.

\section{APPENDIX}

Points of Subjective Equality (PSEs) As a Function of KOR Type, First Versus Second Block of Trials, and Ensemble

\begin{tabular}{llccc}
\hline KOR Type & Block & Ensemble & PSE & $S D$ \\
\hline Arithmetic & first & 1 & 24.72 & 3.19 \\
Arithmetic & first & 2 & 30.62 & 3.34 \\
Arithmetic & first & 3 & 37.04 & 3.26 \\
Arithmetic & first & 4 & 41.89 & 5.25 \\
Arithmetic & second & 1 & 25.11 & 2.42 \\
Arithmetic & second & 2 & 30.88 & 2.44 \\
Arithmetic & second & 3 & 35.90 & 3.71 \\
Arithmetic & second & 4 & 41.94 & 4.16 \\
Harmonic & first & 1 & 24.84 & 4.17 \\
Harmonic & first & 2 & 31.24 & 3.93 \\
Harmonic & first & 3 & 37.61 & 5.30 \\
Harmonic & first & 4 & 44.57 & 7.00 \\
Harmonic & second & 1 & 23.96 & 4.16 \\
Harmonic & second & 2 & 29.91 & 4.27 \\
Harmonic & second & 3 & 35.24 & 5.03 \\
Harmonic & second & 4 & 40.63 & 5.62 \\
RMS & first & 1 & 27.01 & 3.56 \\
RMS & first & 2 & 33.51 & 4.02 \\
RMS & first & 3 & 39.69 & 4.60 \\
RMS & first & 4 & 46.68 & 5.65 \\
RMS & second & 1 & 26.37 & 3.32 \\
RMS & second & 2 & 31.77 & 3.46 \\
RMS & second & 3 & 38.02 & 4.52 \\
RMS & second & 4 & 42.78 & 5.73 \\
\hline
\end{tabular}

(Manuscript received August 26, 2008;

revision accepted for publication October 29, 2008.) 